\title{
EXPERIENCIAS DE TRABAJO BAJO LA NUEVA GESTIÓN PÚBLICA EN CHILE. ANÁLISIS DE LOS SIGNIFICADOS ATRIBUIDOS A LOS PROGRAMAS DE MEJORAMIENTO DE LA GESTIÓN (PMG) POR PROFESIONALES DEL SECTOR PÚBLICO
}

\author{
WORK EXPERIENCES UNDER NEW PUBLIC MANAGEMENT \\ IN CHILE: ANALYSIS OF THE ATTRIBUTED MEANINGS TO \\ THE PROGRAMS FOR MANAGEMENT IMPROVEMENT (PMG) \\ BY PUBLIC SECTOR PROFESSIONALS
}

\section{MARÍA ISABEL LEAL LLANOS}

Universidad Diego Portales, Chile

\section{ANTONIO STECHER}

Universidad Diego Portales, Chile

*Autor de correspondencia:

Antonio.stecherqudp.cl
ÁLVARO SOTO ROY

Universidad Alberto Hurtado, Chile
El artículo busca contribuir al análisis de los procesos de modernización del Estado y reforma a la Gestión Pública implementados en Chile bajo el paradigma gerencial de la Nueva Gestión Pública (NGP). Se presenta los resultados de una investigación psicosocial de corte cualitativo, cuyo objetivo fue describir y comprender los significados que trabajadores profesionales del sector público asignan a los PMG, uno de los principales instrumentos de gestión dentro del trabajo de los servicios públicos en Chile. Se realizaron 12 entrevistas semiestructuradas en un Servicio Público en Chile buscando conocer los sentidos y experiencias de trabajo en torno a los PMG. Los resultados dan cuenta de 8 grandes significados en torno a los PMG, los que dan luces del carácter tensionado, ambivalente y problemático de las experiencias laborales de los profesionales del sector público en el marco de los nuevos sistemas de gestión. Se discuten las implicancias de los hallazgos en relación a los debates actuales sobre los límites e implicancias precarizantes de las reformas gerenciales implementadas bajo el paradigma de la NGP.

Palabras Claves: Nueva Gestión Pública, Chile, PMG, Experiencias laborales, Precarización laboral
The article seeks to contribute to the analysis of the processes of state modernization and public management reform implemented in Chile under the managerial paradigm of New Public Management (NPM). It presents the results of a qualitative psychosocial research aimed at describing and understanding the meanings that professional workers in the public sector assign to the PMG, one of the main management instruments within the work of public services in Chile. Twelve semi-structured interviews were carried out in a public service in Chile in order to understand the meanings and work experiences related to the PMGs. The results reveal eight main meanings of the PMGs, which shed light on the tense, ambivalent and problematic nature of the work experiences of public sector professionals in the framework of the new management systems. The implications of the findings are discussed in relation to current debates on the limits and precarious implications of managerial reforms implemented under the NPM paradigm.

Keywords: New Public Management, Chile, PMG, Labour experiences, Labour Precarisation 


\section{INTRODUCCIÓN}

Al igual que en distintos países de América Latina, el Estado de Chile implementó desde mediados de los años 1990 importantes reformas a la administración pública. Estas diversas reformas de modernización del Estado estuvieron orientadas por el paradigma gerencial de la Nueva Gestión Pública (NGP) y supusieron significativas transformaciones a las formas tradicionales previas de organización del Estado y de sus lógicas de acción pública (Arellano-Gault, 2002; CLAD, 1998; Doña, 2006; Dussauge, 2009a, 2009b; Pliscoff-Varas 2017).

Las reformas, activamente promovidas desde distintos organismos internacionales (BM, FMI, OECD), procuraban modernizar la Administración Pública Tradicional a partir de la incorporación dentro de lo público de una racionalidad económica y de herramientas de gerenciamiento propias de la empresa privada (Arellano-Gault, 2002; Hood, 1991; Guerrero, 2009; O’Flynn, 2007; Osborne \& Gaebler, 1992). Entre otras características, este paradigma se ha caracterizado por la importancia dada a aspectos tales como las capacidades gerenciales, el control de gestión, la medición de resultados y su vinculación a la asignación presupuestaria, los sistemas de incentivos y de renta variable, la creación de mercados y espacios de competencia, la descentralización organizacional y la externalización hacia privados, la separación de las funciones de diseño y regulación (Estado) de las de implementación de programas (privados), el despliegue de nuevas herramientas e instrumentos de gestión de corte empresarial, y la diseminación de nuevos valores y sentidos del trabajo neo-manageriales ligados a la rentabilidad, competencia, calidad y eficiencia, entre otros (Dussauge, 2009a, 2009b; García, 2007; Guerrero, 2009; Pliscoff-Varas 2017). La promesa de la NGP era que con dichas reformas los gobiernos lograrían dar un salto de eficiencia, calidad y transparencia en sus administraciones públicas, lo que resultaba prioritario en contextos socio-económicos de fuertes restricciones presupuestarias, creciente complejidad de la vida social y altas demandas ciudadanas (Arellano-Gault, 2002; Dussauge, 2009a, 2009b).

Los procesos de implementación de la NGP en Chile y América Latina han dado lugar a diversas investigaciones y a un amplio debate respecto a sus variaciones en distintos países, a su efectiva profundidad y alcance, y a sus consecuencias colaterales negativas en términos de precarización y pérdida de sentido del trabajo público. Asimismo, se han discutido extensamente las particularidades de la NGP dentro de una región en que nunca se consolidó previamente un Estado weberiano al modo europeo, sus distintas fases u oleadas de implementación ocurridas desde los años 1990, así como su vínculo con el modelo de desarrollo neoliberal y sus lógicas de privatización y mercantilización ICLAD, 1998; Dussauge, 2009a, 2009b).

Este artículo busca aportar a este campo de discusión abordando, desde la Psicología Social del Trabajo, un ámbito habitualmente menos considerado en los estudios sobre el Estado y la Gestión Pública, referido a los significados del trabajo y las experiencias de los trabajadores de servicios públicos insertos en organizaciones modernizadas bajo los principios de la NGP.

El artículo presenta los resultados de un estudio cualitativo situado en una perspectiva crítico-interpretativa de investigación social (Krause, 1995; Thompson, 1998) que tuvo como objetivo describir y comprender los significados que trabajadores profesionales de un servicio público del Estado de Chile otorgan a un particular 
instrumento de gestión -Programas de Mejoramiento de la Gestión (PMG)-, el cual ha sido desde el año 1998 una de las herramientas centrales del paradigma gerencial de la NGP (Morales, 2014). El análisis de las reformas y procesos de modernización del Estado han tendido a privilegiar ya sea los aspectos organizativos, o de eficacia y productividad, o han instalado discusiones críticas generales respecto al carácter ideológico y político neoliberal de estos procesos. El artículo, así, es relevante en tanto aborda dimensiones psicosociales o subjetivas de los trabajadores de lo público que han sido menos estudiadas, atendiendo a las experiencias, vivencias y significados de dichos trabajadores cuyo trabajo cotidiano se ha visto fuertemente reorganizado por las innovaciones gerenciales de la NGP. Recuperar y conocer esas experiencias resulta clave para una comprensión más integral de los procesos de reforma al Estado, y para aportar a mejores prácticas a nivel de los equipos de gestión y desarrollo de las personas en el sector público. Prácticas capaces de promover tanto la eficiencia de las organizaciones, como el bienestar y la participación de los trabajadores.

\section{NUEVA GESTIÓN PÚBLICA EN CHILE Y PMG}

El desarrollo en Chile de reformas modernizadoras orientadas por el marco de la NGP, iniciado tempranamente en los años 90', ha sido considerado como uno de los más ambiciosos, sistemáticos y profundos en América Latina (Bressner, 2002; Dussauge, 2009). A partir del gobierno de Frei (1994-2000) con la creación del Comité Interministerial de Modernización de la Gestión Pública el año 1994 y, con la publicación del Plan Estratégico de Modernización de la Gestión Pública el año 1997, se inició un proceso para transformar la admi- nistración y gestión de los servicios públicos (Bressner, 2002; Ramírez, 2004; Pliscoff-Varas, 2017). El foco de las reformas era la calidad de los servicios públicos, la eficiencia y efectividad en el uso de recursos, la mejora de la experiencia para los usuarios y la introducción de TICs. Con ello se buscaba no sólo responder al desafío de fortalecer las capacidades del aparato público, sino también a las exigencias y recomendaciones de organismos internacionales, así como a una nueva serie de demandas provenientes de la sociedad civil a las cuales el Estado debía adaptarse (Bressner, 2002; Ramírez, 2004). Junto al Plan Estratégico de Modernización de la Gestión Pública del año 1997 - el cual implicó mejoras en los sistemas de planificación, de control de la gestión y de medición de indicadores de desempeño - PliscofVaras (2017) destaca otros dos hitos centrales de la modernización del Estado en Chile: “...la instalación de un sistema de evaluación y control de gestión por parte de la Dirección de Presupuestos (...), el cual se instala el 2001, y la creación del Sistema de Alta Dirección Pública en 2003, que empieza a funcionar en 2004, en el marco de la creación de la Dirección Nacional de Servicio Civil." (p.144).

Las reformas de la gestión pública en Chile se desarrollaron como un proceso gradual y acumulativo, al que se iban sumando progresivamente nuevos servicios, y cuya implementación supuso la aprobación de distintos proyectos de ley en el marco de complejas negociaciones y disputas con la oposición y los gremios de los trabajadores públicos (Morales, 2014). PliscoffVaras (2017) plantea que son tres los principios e innovaciones organizativas dominantes en el proceso de implementación de la NGP en Chile, cada una de las cuales implica una ruptura con la matriz previa de la administración burocrática. 
La primera de ellas es la contractualización, que refiere a la dinámica a través de la cual el Estado externaliza la ejecución y administración de bienes y servicios públicos a partir de la subcontratación y establecimiento de contratos con empresas privadas, con las que se configuran redes de acción Público-Privadas (APP) encargadas de la acción pública (Pliscoff y Araya, 2012; Devlin y Moguianski, 2010). Estas redes permitirían reducir costos al Estado, incrementar la calidad de los servicios con empresas especializadas y separar las funciones de diseño, monitoreo y evaluación (Estado) de aquellas de ejecución propiamente tal del servicio (privados). La instalación de estas redes surgidas de APP presentó en los primeros años dificultades, casos de corrupción y múltiples tensiones, dado que el Estado perdía control sobre las acciones de los privados y no contaba con el personal suficiente para supervisar y fiscalizar (Pliscoff y Araya, 2012; Pliscoff-Varas, 2017). Gradualmente se fueron instalando una serie de instrumentos de acción pública más sofisticados -contratos, convenios, bases de licitación y de concursos- que permiten gobernar, protocolizar y controlar la acción de privados, volviéndose en la última década mecanismos fundamentales para el desarrollo de la política pública (Soto, Fardella, Valenzuela y Carvajal, 2017).

La segunda innovación fue la instalación de una gerencia pública profesional, la que ha sido quizás una de las reformas más visibles y eficientes en el proceso de modernización del Estado de Chile (Bressner, 2002; Morales, 2014; Pliscoff-Varas, 2017). El objetivo era superar las trabas burocráticas de la gestión pública, proveyendo al Estado con servidores públicos altamente cualificados y profesionales a los cuales otorgar una mayor discrecionalidad para la toma de decisiones.
A partir de la creación del Sistema de Alta Dirección Pública (SADP) a inicios de los 2000 se logró aumentar las competencias gerenciales de los servicios públicos, mediante procesos de selección de gerentes públicos basados en la meritocracia y la transparencia, siendo un sistema reconocido a nivel Latinoamericano (Bressner, 2002) y el que ha logrado un impacto en la eficiencia en el uso de recursos públicos (Morales, 2014).

En tercer lugar, Pliscoff- Varas (2017) destaca innovaciones orientadas a lograr una gestión presupuestaria flexible vinculada a resultados e incentivos asociados, lo que implicó la generación de asignaciones de renta a los funcionarios públicos en función del desempeño y cumplimiento de objetivos de modernización de los distintos servicios. Morales (2014) señala que al año 2010 el sistema de Evaluación y Control de Gestión -que vincula la asignación y uso de recursos a los resultados y desempeño alcanzados por los servicios- estaba compuesto por cuatro instrumentos: (i) Balances de gestión integral; (ii) Evaluaciones de Programas Gubernamentales; (iii) Indicadores de desempeño institucional (IDI) y (iv) formatos de presentación de iniciativas al presupuesto; y tenía tres mecanismos de incentivo salarial al desempeño: (i) Los programas de mejoramiento a la gestión (PMG), (ii) la ley médica y (iii) las metas de eficiencia institucional. Morales (2014) señala que estos mecanismos de control y gestión basado en el ciclo objetivo/desempeño/medición/incentivo han mostrado resultados discretos en términos de eficiencia y efectividad, y en ocasiones han sido desvirtuados de su sentido inicial al promover conductas estratégicas orientadas a la obtención de la recompensa, más que a la mejora de la gestión y el servicio público (Pliscoff, 2018; Morales, 2014). 
Los Programas de Mejoramiento de la Gestión (PMG), que han operado como uno de los principales mecanismos de incentivo salarial al desempeño dentro del nuevo ecosistema de la Nueva Gestión Pública, tienen su origen en la Ley No 19.553 del año 1998 (Gobierno de Chile, 2014, 2018). A partir de 2012 el incentivo monetario a los y las trabajadores corresponde a un aumento de un $7,6 \%$ de las remuneraciones en caso que la institución alcance más de un $90 \%$ de cumplimiento de sus metas anuales, o un aumento de un 3,8\% si dicho cumplimiento fuere igual o superior al $75 \%$ e inferior a $90 \%$ (Dipres, 20201)1. Dichos incentivos monetarios se pagan trimestralmente al año siguiente del cumplimiento de los objetivos anuales comprometidos.

Como se señala en la página Web de la Dirección de Presupuesto del Gobierno de Chile, desde el año 2014, los PMG cubren un total de 194 instituciones y más de 87 mil funcionarios, constituyéndose en el mecanismo de incentivo de remuneraciones más importante dentro de la administración pública de Chile (Dipres, 2021).

Los PMG son coordinados por un Comité Triministerial integrado por los Ministerios del Interior, Hacienda y Secretaría General de la Presidencia. Este Comité Triministerial tiene dentro de sus principales funciones aprobar y evaluar los compromisos de los Servicios. Por su parte la Dirección de Presupuestos (Dipres) cumple las funciones de Secretaría Técnica (Dipres, 2021).

Desde el año 2000 los PMG se organizan a partir de la definición de un conjunto de áreas comunes que son transversales a todas las instituciones públicas, y las cuales se han ido ampliando y precisando en función

\footnotetext{
1 El año 2014 se redujo el piso mínimo de cumplimiento para cada indicador de $95 \%$ a $75 \%$, existiendo incentivo a niveles de logro inferiores al 95\%, proporcionalmente, y hasta un nivel de $75 \%$
}

de las distintas evaluaciones que se han hecho de dicho instrumento (Banco Mundial, 2008; Dipres, 2021). Como referencia, es posible señalar que al año 2010 los PMG se organizaban con base a trece sistemas, doce centrados en procesos y uno referido al sistema de Planificación y Control de Gestión de cada Servicio. Los sistemas referidos a proceso eran: Capacitación, Higiene-Seguridad y Mejoramiento de Ambientes de trabajo, Evaluación del Desempeño, Sistema integral información y atención ciudadana y Sistema de acceso a la información pública, Gobierno electrónico-Tecnologías de la información, Auditoría interna, Gestión territorial, Compras y contrataciones del Sector Público, Administración Financiera y contable, Enfoque de género, y Sistema de gestión de la calidad (ISO) (Dipres, 2021).

En lo que respecta a las metas e indicadores anuales de los PMG, en las últimas versiones de la ley 19.553, estos deben vincularse a la misión institucional, objetivos estratégicos y productos significativos de cada ministerio o servicio. Asimismo, deben haber sido validados en el sistema de planificación y control de gestión del Programa de Mejoramiento de la Gestión y quedar establecidas en un convenio de desempeño que firman los servicios con el respectivo ministro, en el último trimestre de cada año (Biblioteca del Congreso, 2021). El cumplimiento de las metas establecidas incorpora mecanismos de consulta y entrega de información a las asociaciones de funcionarios de los Servicios (Biblioteca del Congreso, 2021). Por su parte, el cumplimiento de las metas es verificado por la unidad de auditoría interna de cada servicio y ministerio, las que a su vez reportan a las plataformas correspondientes de la Dirección de Presupuesto. Posteriormente, y a partir del año 2011, la validación técnica del cumplimiento de los PMG es 
realizada por expertos externos al Ejecutivo. Dichos expertos son contratados vía de licitación pública, buscando resguardar la independencia y transparencia del proceso (Dipres, 2021).

En la medida que los servicios o ministerios cumplen los indicadores transversales de algún PMG en un alto porcentaje, por un tiempo sostenido, y sobre el estándar que la Dipres determina para cada caso, existe la posibilidad de que puedan egresar o ser liberados del cumplimiento de esas metas transversales. Ello a diferencia de aquellos indicadores específicos de los PMG, que se vinculan al giro o foco de actividad de cada servicio. En el caso de éstos últimos, si se supera el estándar y se quiere abandonar una medición esta debe ser reemplazada por otra.

Por último, los PMG contemplan aquellas situaciones consideradas "externas" que eximen del cumplimiento de los PMG a los Servicios, como aquellas vinculadas a las reducciones presupuestarias externas al Servicio, hechos fortuitos comprobables, catástrofes y cambios en la legislación vigente (DIPRES, 2021).

\section{METODOLOGÍA}

La investigación asumió una perspectiva interpretativa y contempló un diseño metodológico cualitativo, orientado a reconstruir y comprender los significados que profesionales de un servicio público en Chile asignan a los Programas de Mejoramiento a la Gestión (PMG) (Krausse, 1995).

Junto al paradigma interpretativo, el diseño asumió ciertos aspectos de la perspectiva crítica presentes en la Psicología Social del Trabajo (Stecher, 2010; Thompson, 1998), la cual permite vincular la producción de sentidos que realizan los actores sociales en sus contextos cotidianos a las estructuras, instituciones y contextos históricos específicos. En el caso de este estudio, lo anterior supuso poner en relación los sentidos sobre los PMG presentes en los relatos de los entrevistados, con los procesos histórico-institucionales de modernización del Estado y reforma a la Gestión Pública acontecidos en Chile en las últimas 3 décadas.

Los participantes del estudio fueron 12 profesionales empleados en modalidad a contrata en un Servicio Público del nivel central del Estado de Chile, en el cual los PMG cumplen un importante rol en la organización de los procesos de trabajo. Los profesionales entrevistados tienen entre 35 y 65 años y cuentan con entre 5 y 35 años de experiencia laboral en el Estado. Nueve son mujeres y tres son hombres y en términos de campos profesionales dos de ellos se sitúan en las Ciencias Sociales y los otros 10 en distintas ramas de la ingeniería.

Las técnicas de producción de la información fue la entrevista individual semiestructurada (Flick, 2004). Se utilizó un guion de entrevista que contempló las siguientes dimensiones: trayectoria laboral, rol en la organización y desempeño del trabajo cotidiano, función y lugar de los PMG en la organización de sus procesos de trabajo, percepción del trabajo público y de sus transformaciones bajo la lógica de los PMG.

La recolección de los datos y el análisis de los mismos se realizó durante el segundo semestre del 2018 y estuvo a cargo de la autora principal del artículo. Todas las entrevistas consideraron la firma de una carta de consentimiento informado -aprobada por un comité de ética de investigación de una Facultad universitaria- donde se explicitaron los objetivos del estudio y se garantizó tanto la confidencialidad como el anonimato de la información. Las entrevistas duraron alrededor de 60 minutos, fueron grabadas y luego transcritas para el análisis. 
La estrategia de análisis de la información fue el análisis de contenido de orientación cualitativa (Ruiz, 2003), considerando para el proceso de codificación de las entrevistas algunas de las orientaciones y criterios de la codificación abierta de la teoría fundada (Corbin \& Strauss, 1998). A través de sucesivas fases de lectura y codificación se fue elaborando una matriz interpretativa compuesta por los principales significados que los profesionales entrevistados asignan a los PMG, al modo como estos configuran sus experiencias laborales y a la manera en que dichos sentidos y experiencias se vinculan con las nuevas lógicas de organización de la NGP. Más específicamente, los pasos del análisis del material fueron los siguientes:

* Transcripción de las entrevistas.

* Lectura en profundidad de cada entrevista para construir una primera idea general de la trayectoria y experiencia laboral de cada entrevistado.

* Codificación de las entrevistas, partiendo por las de los trabajadores con mayor antigüedad, para la generación de categorías de análisis que daban cuenta de los principales significados con que los PMG aparecían en los relatos de los trabajadores.

* Se analizaron nuevamente las entrevistas en base a estas categorías formando así un esquema general con las categorías y sus relaciones, buscando generar una comprensión compleja del objeto de la investigación.

* Se propuso una interpretación respecto a las principales tensiones presentes en las experiencias laborales vinculadas a los PMG, estableciendo relaciones entre estas y la revisión de antecedentes sobre la NGP en Chile.

\section{RESULTADOS}

A partir del proceso de codificación y análisis realizado se identificaron en los relatos de los profesionales 8 sentidos transversalmente compartidos en torno a los PMG. Estos significados se superponen entre sí, incluso dan cuenta de contenidos que podrían considerarse contradictorios, y de esta manera dan cuenta de la complejidad y el dinamismo de las experiencias subjetivas del trabajo enmarcado en los PMG. Se describen e ilustran en este apartado dichos sentidos, dando cuenta, ahí donde es pertinente, de ciertas diferencias en función de variables como edad, antigüedad y sexo de los profesionales.

Los PMG como una herramienta de gestión moderna, eficiente y transparente pero con tensiones y falta de recursos en su implementación.

Un primer sentido que surge del análisis se refiere a los $P M G$ como una herramienta que contribuye al alcance de la eficiencia y la transparencia, considerados valores claves en los procesos de modernización del Estado. Más allá de las específicas valoraciones, se aprecia un cierto sentido compartido de que los PMG han permitido organizar, homogenizar y planificar el trabajo bajo ciertos preceptos que apelan a la instalación de una cultura de la eficiencia, la evaluación y la mejora continua, facilitando y permitiendo visibilizar resultados concretos de la calidad del trabajo.

Lo anterior, es posible observarlo en el siguiente relato:

"Los PMG te permiten ordenar, clasificar, pero más bien aquí la Dipres buscó una forma de medir a todos por igual, porque imagínate la mole de Estado y esta era una forma concreta, pero me acuerdo una vez que el asesor 
ministerial alguna vez dijo, nosotros creamos fórmulas pero ustedes tienen que traducirlas en fácil, pero qué es eso...yo no sé si aquí es igual o si en Hacienda, no creo po, no podís medir a todos por igual, pero también entiendo que se debe ordenar y demostrar que trabajamos, jajajajajaja, no pero fuera de bromas, igual es bueno mostrar que se intentan hacer las cosas bien, eso apunta a transparencia también" (Mujer, 59 años)

En este sentido, entendidos como herramienta de gestión moderna y eficiente, los PMG aparecen como garantes de lo que implica ser un buen servicio público y por ende un buen trabajador. Sin embargo, más allá de ese reconocimiento general, los profesionales identifican múltiples tensiones en la implementación concreta de los PMG en su Servicio. Muchas veces no se cuenta con los recursos y soportes para cumplir las metas definidas, se produce una responsabilización sobre ciertas personas o equipos sino se alcanzan los objetivos, y se produce una alta presión por el cumplimiento de metas desde las jefaturas y los pares, dado los bonos asociados a los PMG, orientándose todo el trabajo en el servicio por la lógica de la meta y el bono. El siguiente fragmento de una entrevista apunta a lo anterior:

“Mira si al final uno asume no más, hace lo que puede, y más, porque si no se cumple el PMG tamos todos fritos, pero por el bono, lo peor que te puede pasar porque si no cumplimos el que estaba a cargo pasa a la guillotina de todos, (...) es raro bien raro eso, no sé cuándo la gente va entender que tenemos todos que trabajar pal bono, o sea el PMG (...)...mira yo tengo claro que debo trabajar para estos procesos, cada vez más nos piden más cosas, te doy un ejemplo la seguridad de la información responden a un sinfín de indicadores, pero no tenemos ni uno para comprar un software que nos facilite la pega y (...) se pueda cumplir con los requerimientos de ese PMG, entonces la jefatura de servicio nos plantea innoven, sean creativos, armen algo con lo que hay, pero trabajamos en la pobreza sobre todo ahora que la reducción presupuestaria nos acecha" (Hombre, 44 años).

Esa tensión entre, por un lado, una valoración de los PMG como mecanismos de la necesaria modernización del Estado, y que contribuyen a definir procesos, establecer metas, transparentar desempeños y optimizar la asignación de recursos, y, por otro lado, la percepción de que su implementación concreta en distintos servicios está llena de tensiones y dificultades, constituye un sentido transversalmente compartido por los entrevistados. El siguiente fragmento ilustra también esa tensión:

“Mira tengo dos miradas: la mirada pura por decirlo así, creo que los PMG ayudan mucho ayudaron mucho al proceso de mejoramiento de la gestión a poner en el tapete el tema de la modernización del Estado ya por eso entre la teoría propiamente tal considero que ayuda, el tema es que como llevan a cabo el PMG, creo que hace que el proceso se dificulte y vuelvo a repetir y si la gente a cargo del PMG fuera una unidad potente con buena habilidades y buenas competencias te podría facilitar muchas cosas porque hay muchas cosas, que uno como encargada de PMG tienes que buscarla tu solita y en la marcha, entonces si tuvieran claro si al principio con instrucciones claras con todos los insumos e información (...) .....ahí en la teoría es bueno pero en la práctica creo que se complejiza más por la forma" (Mujer, 35 años). 


\section{Los PMG como parte de un proceso de control e intensificación del trabajo público.}

En segundo lugar, los PMG aparecen significados como herramientas que han implicado una intensificación y un mayor disciplinamiento en el trabajo. Las exigencias de precisión, reportabilidad, la presión del bono trimestral, el control, los indicadores de desempeño, la entrega de resultados en tiempo record teniendo que completar labores fuera de la jornada regular, la vigilancia de los compañeros, entre otros, se han ido transformando en elementos que de manera silenciosa han precarizado la experiencia de trabajo de estos profesionales en el Estado. El siguiente relato de entrevista da cuenta de esta idea:

"Para mí quedarse más tarde es intentar hacer las cosas bien y terminarlas en los plazos que se requieren, pero si la necesidad del servicio es estar las 24 horas bueno uno tiene que cuidar la pega también y si hay que quedarse nos quedamos no más, es como que es bien visto por los jefes quedarte más tiempo (...) pero nunca me alcanza la jornada sobre todo cuando hay que reportar porque eso no te quita el tiempo destinado a tus otras actividades, tay frito no más, te tienes que quedar y terminar, no se te puede pasar un detalle porque una equivocación y se te viene todo el mundo encima cachai que todos tienen derecho a decir, opinar de tu pega y no tienen idea" (Mujer, 49 años).

Es posible observar, en este sentido, el modo en que los PMG instalan lógicas de autovigilancia, autoexigencia y vigilancia de pares que pueden configurar un entorno de trabajo de mucha presión, muchas veces de carácter solitario y donde se tensionan los vínculos de colaboración.
"Mira a mí los PMG me generan varias cosas, si bien yo creo que sirven, pero fíjate que yo me las arreglo solita para que esté toda la información para generar el informe y entregar los medios de verificación cuando corresponde, igual yo prefiero eso porque me aseguro que la información esté cuando tiene que estar pero para tener esa seguridad me hago cargo sola con todo lo que eso significa (...) pero anda a saber si uno se equivoca porque el castigo es grande" (Mujer, 35 años).

En los relatos, son los trabajadores mayores y de mayor antigüedad dentro del servicio los que más resienten la presión, debido en parte a la exigencia adicional que supone adaptarse a las nuevas tecnologías y plataformas a partir de los cuales se gestionan los PMG.

" no yo prefiero trabajar sola, al menos en lo que puedo, los cabros jóvenes puro que te critican... uno se da cuenta porque en temas tecnológicos soy mas lenta pero aunque me demore más lo hago, imagínate cuando tenemos que hacer el reporte trimestral yo me he tenido que quedar aquí sola con las ánimas hasta las 11 de la noche" (Mujer, 62 años).

Los profesionales más jóvenes, cuya inserción laboral se produjo ya bajo el marco de la NGP, si bien reconocen la exigencia y presión que suponen los PMG, tienden a naturalizarla y normalizarla, destacando incluso que dicha presión es algo que los ayuda a funcionar mejor.

El siguiente fragmento ilustra esta idea:

"A ver, mira, los PMG uno los reporta, en todo caso yo empecé a trabajar en el servicio público bajo este sistema no conozco otra forma, si puedo ver cómo han ido cambiando en el camino, cada vez aparecen más cosas, pero uno tiene que entregar productos y esos generalmente son los 
reportes que los construyes durante el año, pero siempre se dejan para el final, pero eso es porque en todo caso se funciona mejor así o sea yo funciono mejor bajo presión, genero mejores informes, cuando estoy relajada como que no me salen las cosas, siento que me disperso, igual uno está haciendo su trabajo de hecho es raro cuando no te presionan de otros departamentos por la información, o las áreas técnicas, así que al final uno se acostumbra" (Mujer, 35 años).

Por último, es importante consignar que en algunos relatos se indica que existiría una generización del trabajo en torno a los PMG, siendo que serían las profesionales mujeres las que reciben la responsabilidad de gestionar los PMG en los servicios públicos, con la consecuente responsabilización y sobrecarga que ello implica.

“Si te das cuenta gran parte de las que llevamos el tema o a cargo de los PMG, somos más Mujeres, también en el área de control de gestión donde se entrega todo al menos aquí son Mujeres, en la Dipres las que llevan históricamente el tema de las MEI y PMG son Mujeres, y así suma y sigue, en otros servicios habrán más [Hombres] no lo sé, pero uno va a las reuniones técnicas del nivel central donde vamos todos los servicios siempre somos más vistas las Mujeres en estos temas, bueno puede ser casualidad, pero en mi experiencia los Hombres llevan los cargos importantes y los técnicos, y nosotras el detalle y te pongo un ejemplo en Fiscalización hay varios chiquillos, pero la que lleva el orden del presupuesto, de los indicadores, de los detalles es una Mujer" (Mujer, 59 Años).

\section{Los PMG como mecanismo y derecho adquirido de mejora de las remuneraciones.}

Un tercer significado que emerge del análisis de los relatos de estos trabajadores acerca de sus experiencias labores con los PMG, es su consideración como mecanismos que incrementan las remuneraciones a partir de bonos trimestrales que son considerados como un derecho adquirido, y no tanto, o no centralmente, como una herramienta para mejorar la calidad de los servicios públicos en general. Existe, de este modo, una valoración de los PMG en tanto posibilidad de disminución de lo que se considera una brecha histórica salarial en el Estado.

Aquellos profesionales directamente a cargo de gestionar los PMG, suelen mirar críticamente esta forma de significar los $P M G$ en que estos quedan desacoplados de su función original de asociar bonos a cumplimiento de metas, aunque comparten que existe un sentido común compartido de que dichos incentivos son ya parte regular del sueldo.

"Bueno todos sabemos que los bonos son los bonos, que vienen cada tres meses, lo que el resto del equipo no considera es que si no cumplimos con los requerimientos chao bono, igual lo ganamos siempre entonces a mí no me complica el tema de las metas, porque siempre se hará lo que sea para informar, igual si nos quitan esas lucas es como si nos bajaran el sueldo, por eso yo creo que los PMG ya no deberían considerarse como parte de ese beneficio"(Hombre, 47 años).

\section{Los PMG como exigencia de nuevas competencias genéricas en gestión de instrumentos.}

Un cuarto sentido que emerge se refiere a cómo el trabajo público bajo la lógica de los PMG exige a los 
profesionales nuevas competencias y capacidades, las que muchas veces son muy distintas y distantes a las de su formación profesional de origen. Al respecto, se señalan -especialmente para aquellos cargos directamente responsables de un PMG- capacidades de orden, planificación, monitoreo del trabajo de otros, gestión de información, manejo de plataformas, registro de desempeños, autonomía y flexibilidad. Estos nuevos perfiles de cargo se expresarían en los procesos de reclutamiento y selección del Estado, donde se solicitan estas competencias con un fuerte énfasis en la capacidad de ser flexible para saber afrontar las exigencias de adaptación al cambio constante. Los siguientes fragmentos ilustran este punto:

"Yo soy parte de la Unidad de control de gestión y mi rol es auditar que los PMG se cumplan, pero esto a veces es ingrato porque tus compañeros no entienden la presión que tenemos, ellos creen que uno los anda persiguiendo para acusarlos y no es así, es parte de la exigencia que como unidad tenemos" (Mujer, 59 años).

“en mi caso he estado a cargo de varios de los cuales no necesariamente responde a mi área técnica, pero como soy ordenado y siempre digo que sí me toca, pero igual es complejo sobre todo cuando tienes que reportar el trabajo de otros, que sabis tú si esa información que recibes se hizo con la claridad o con la calidad que amerita un proceso y ahí uno confía no más, pero también pones el pellejo, entonces ahí yo te digo que se da eso de cumplimiento".

De algún modo, la expansión de los PMG exige a los profesionales aprender un nuevo lenguaje y nuevos códigos, volverse especialista en los instrumentos de gestión, reorganizar o incluso alejarse de su rol profesional considerando aquellas competencias para la gestión y monitoreo de los PMG, los que vienen definidos desde el nivel central del Estado.

\section{Los PMG como mecanismos que externalizan y alejan de los espacios locales la definición y evaluación de los objetivos de trabajo}

Los PMG configuran una experiencia de trabajo donde los objetivos del trabajo y su evaluación queda situada en una instancia distante y externa, que muchas veces desconoce el trabajo real de cada servicio, y la que en ocasiones inclusive puede corresponder a consultoras privadas que no son parte del Estado. En este punto, la DIPRES juega un rol central en la planificación y formulación del programa marco de los PMG, y en su posterior monitoreo, control y evaluación del logro de resultados.

Muchas veces dicha evaluación supone la intervención de una consultoría externa para su revisión y sanción final. De este modo, los profesionales del servicio no tienen una interacción directa con quienes formaron parte de la formulación y evaluación final del proceso, solo la tienen con los departamentos de control de gestión de sus propios servicios, cuya función es visar y poner a disposición la información, a través de plataformas de reportabilidad, a la DIPRES y a eventuales consultoras externas. Son estas instancias las que definen finalmente si los objetivos de un PMG han sido cumplidos y pueden ser aprobados.

En esta cadena de control y evaluación los profesionales solo generan información, lo que en la mayoría de los casos es fuente de confusión y de estrés, ya que los agentes revisores externos no tienen mayor conexión con los desafíos y dificultades del trabajo real realizado por los servicios públicos. Se produce una dinámica 
que puede llevar a una pérdida de sentido del trabajo público, sometido a una lógica mecánica de reportes de información a unidades externas.

“Mira yo reporto información a 3 unidades distintas, a la hora de los kiubo no sé quién tiene la razón por eso yo solo cumplo con entregar lo que me corresponde, pero igual es raro porque mi jefatura directa no se mete y no sé si le interesa, pero alguien dijo que yo tenía que hacerme cargo de esa información, tampoco sé si alguien le importa y si eso cuenta en mi evaluación de desempeño individual, porque igual a veces tengo que hacer mi pega específica y esto" (Mujer, 45 años).

“Al final está claro que tus esfuerzos por contribuir se pueden ir a la borda, porque tú sabes que quienes revisan y aprueban son otros personajes del mundo privado, consultoras externas, pero crees tú que saben lo que significa trabajar en un servicio público, lo que significa fiscalizar, atender público, cumplir con lo que se pide, o simplemente de que se trata lo que hacemos nosotros... a mí eso me molesta, pero hasta ahora no hemos tenido fracasos, pero sí hemos tenido que ajustar cosas a la pinta de otros que no saben, entonces de qué estamos hablando, nos piden A y nosotros sabemos que es $B$, pero como lo dicen ellos tenemos que poner B no más, pero eso no refleja lo que hacemos y lo que significa hacer que las cosas anden, se mantengan, no sé, funcionen" (Mujer, 49 años).

En los trabajadores mayores existe una mayor distancia crítica frente al rol de evaluación de las consultoras privadas, el cual es visto como una anomalía y despilfarro de recursos del Estado. Piensan, recordando tiempos previos, que esos recursos deberían invertirse en capacitar y fortalecer las capacidades de los servicios públicos.
“Yo soy más vieja y llevo muchos años, más de 30 en el Estado y para mí estas consultorías externas que se contratan para validar nuestros indicadores en la Dipres no han hecho más que robustecer a los privados, es un negocio, pero eso lo ve uno que es más vieja porque la gente joven aquí ojalá le externalicen todo, la moda de la consultoría o la flojera ya me confundo a esta altura... ... si esa plata que se gasta que son millones miles se sumara a los servicios tendríamos tal vez otra infraestructura, más equipos, más capacitación, y yo creo que eso si favorecería que el servicio fuera mucho mejor" (Mujer, 59 años).

\section{Los PMG como bloqueo a la innovación y creatividad colectiva.}

Al relatar las experiencias de trabajo con los PMG, los profesionales dan cuenta de un sexto sentido referido a la falta de tiempo y espacio para la creatividad e innovación que suponen los PMG. En tensión con los discursos de modernización del Estado que promueven y realzan el valor de la innovación, los PMG en el espacio laboral son significados como herramientas que, al intensificar y estandarizar el trabajo, limitan muchas veces las posibilidades para pensar y crear colectivamente. Como indica una entrevistada:

"Siento que en la dinámica de la situación pública hay como muy poco tiempo pa' crear, pensar, no tenemos mucho tiempo de ocio, ese bueno que yo le llamo, a veces yo en el bus me doy un momento de ocio pa' pensar pa' crear entonces por eso de repente yo me pongo muy creativa, pero en el bus cuando me vuelvo a [ciudad donde vive] porque en la oficina no puedo... no me da, pero ahí se me ocurren cosas a veces" (Mujer, 35 años). 
Innovar en el Estado aparece como algo altamente complejo, por la rigidez del sistema, por la fragilidad de los espacios, pero también por el constante adelgazamiento del trabajo colaborativo, solidario y de intercambio. Por otro lado, los recursos de infraestructura, económicos, de tecnologías, mayor dotación de personas, por mencionar algunos, son siempre escasos y frágiles, siempre condicionados a los riesgos de las reducciones presupuestarias de los gobiernos.

En este sentido, la trama de indicadores asociados a los PMG aparece no sólo definida principalmente desde fuera y desde arriba, sino que además no ofrece posibilidades efectivas para discutirse o negociarse a la luz de acuerdos o propuestas colectivas desde el nivel local del trabajo público. Como indica la misma profesional:

“Estas cosas constriñen, están demasiados dirigidos no te dejan incorporar algo nuevo o nuevos desafíos, la Dipres te dice proponga indicadores y cuando lo propones te dice no, a través de la plataforma te preguntan, te cuestionan, te dicen los servicios tienen muchos indicadores deben ser menos cuando nosotros decimos este ya cumplió su ciclo o no es de nuestra competencia, nos dicen no es que usted no puede sacar un indicador y si saca uno tiene que poner otro, entonces eso es contradictorio con lo que te habían dicho al principio"(Mujer, 35 años).

Esta ausencia de espacios colectivos para reflexionar, prensar e innovar -dada la falta de tiempo, la intensificación del trabajo y la insuficiencia de recursos- produce una precarización de las experiencias de trabajo, y favorece lógicas de coordinación funcional orientadas por la productividad de las metas inhibiendo otras formas de vínculo colectivo de carácter más colaborativo, participativo y de co-construcción conjunta.

\section{Los PMG, la mecanización y la pérdida de sentido del trabajo público.}

Un séptimo sentido que emerge desde los trabajadores entrevistados respecto a los PMG, dice relación con el modo en que la lógica de los indicadores, el discurso de la calidad y la productividad, la responsabilización individual y los bonos asociados al cumplimiento de metas puede llevar a una cultura orientada a disimular los incidentes, las fallas, los defectos; y donde se pierda de vista el objetivo que tiene un servicio público y el sentido más general del trabajo público. Al respecto, los sujetos entrevistados plantean cuestionamientos a la lógica de cumplimiento e incentivo vigente, pero a la larga se adaptan a ella y buscan cumplir con lo establecido, dado que el Servicio debe responder de alguna manera, y si esto ocurre se mantiene también el trabajo y el nivel de remuneraciones. El siguiente fragmento de entrevista ilustra este punto:

“Encuentro que los PMG y sus bonos son un poco macabros y perversos, no sé si será el minuto para dar mi opinión, pero porque emmm.... es como al final estoy haciendo algo para recibir algo a cambio, no por el sentido, muchas veces perdemos el sentido de por qué tenemos un PMG, porque hay que cumplirlos para ganarnos el bono y cerró ahí, no leemos el trasfondo, no leemos entre líneas, funcionamos como mecánicos y hay que hacer esto y cerró ahí, nunca a lo mejor hemos estudiado o hemos considerado si ese PMG es útil o no, lo considero como que hay que hacerlo y se acabó y por lo mismo te digo no tenemos un tiempo adecuado para pensar, analizar, cuestionar, no sé, pero ese es el tema un PMG está solo relacionado a las lucas por eso creo que es perverso y podría ser mejor cachai" (Mujer, 44 años). 
Los trabajadores del aparato público se refieren al Estado como un empleador deficitario, donde las desigualdades entre distintos trabajadores y unidades se pueden observar claramente. Esto hace que los trabajadores se sientan exigidos no sólo por las demandas actuales de la ciudadanía y usuarios de los Servicios, sino también por un Estado empleador que a través de distintos dispositivos de control y herramientas de gestión exige ciertas metas y opera con un mandato de la probidad. Sin embargo, muchas veces los mismos principios de probidad, transparencia y eficiencia que los entrevistados comparten, entran en tensión con la propia lógica de trabajo que instalan los PMG. Se constata, así, una experiencia de trabajo en que los PMG -en ciertas fases del proceso de trabajo- se asocian a una vivencia de desazón, malestar y sufrimiento en tanto aparecen forzando a los profesionales a alejarse de ciertos valores profesionales o de lo que ellos entienden como el objetivo y razón de ser principal de un cierto servicio público. Esa tensión es compleja de abordar pues, al mismo tiempo, los profesionales reconocen la necesidad de los bonos de desempeño, así como de sus empleos en el Estado como fuente de ingreso y mantención de sus familias. Como se aprecia en los siguientes dos fragmentos:

“...la consigna de base es el cumplimiento [reportar el logro de indicadores] (...) y eso me afecta a veces, porque igual uno tiene otros valores pero que haces si se llega a perder el bono, pero también tengo un master y que hago con eso si al final tengo como parte de mi práctica laboral informar, dar cuenta, que se yo" (Hombre, 44 años).

"mira al final uno hace lo que puede, igual uno intenta hacerlo bien, pero a veces considero el trabajo estresante porque no te puedes equivocar, pero fome porque no logro ver cómo me desarrollo aquí, a veces me dan ganas de irme, pero tú crees que será fácil encontrar trabajo a esta altura si yo llegué aquí como alumno en práctica, después me titulé, hice mi carrera aquí y ya han pasado [más de 20 años] de eso, pero es complejo vivir con presiones de arriba, [nuestro trabajo] no es cualquier cosa tiene que ver con cuidar a otros, pero a veces nos quedamos cortos y que hacemos con eso le damos no más a mí me tirita todo, pero he aprendido a considerar el mal menor, a veces me gustaría hacer las cosas de otro modo, pero no se puede, hay que ajustarse a lo que está definido" (Hombre, 47 años).

Los PMG como elemento que homogeniza y no reconoce la particularidad de cada servicio público y del Estado.

Un octavo sentido de los PMG se asocia a la idea de que la implementación de los PMG opera muchas veces con una lógica gerencial que no considera las particularidades de la administración pública -falta de recursos, estructuras jerárquicas, normativas burocráticas- ni la especificidad sectorial de cada área o servicio. Lo anterior lleva a que no se reconozca la especificidad del trabajo real que enfrenta cada servicio, producto de la lógica de homogenización, estandarización y productividad de los PMG. En este fragmento buscamos ilustrar este punto que tiene un impacto en el clima y la motivación en el trabajo:

“Lo moderno o innovador hace que muchas cosas sean tan estándares, porque eso yo creo que al final atenta como al objetivo de estos instrumentos y para que los servicios funcionemos mejor, pero si están las cosas predeterminadas y todos los servicios tenemos que hacer lo 
mismo, más o menos las mismas cosas, eso a algunos servicios les queda súper grande y les cuesta mucho hacer esos estándares, y es frustrante que te pongan muchas metas que no puedes cumplir y eso afecta la calidad del trabajo, la motivación, clima laboral, porque no es lo mismo por ejemplo hablar de responsabilidad social empresarial en minería por ejemplo que de participación ciudadana en salud, es casi imposible, pero claro un consultor externo tampoco sabe en profundidad la relevancia de las diferencias en cada servicio, si hasta son culturas y organizaciones diferentes...entonces como tú sabes que al final es un externo el que evalúa uno intenta también hablar de responsabilidad social empresarial, por ejemplo, pero tú sabes cuánto se invierte en esto una empresa privada y nosotros con el tema de ciudadanía no tenemos presupuesto, así no alcanzamos el nivel que se requiere" (Mujer, 49 años).

Este intento del Estado por fomentar la eficiencia a través de la homogenización o estandarización en torno al control y evaluación tiene un alto costo para los trabajadores que son llevados de manera constante a adaptarse a situaciones nuevas, entrar en permanentes juegos de ajustes del sistema, aun cuando no se esté de acuerdo con esas instrucciones.

\section{DISCUSIÓN}

Los ocho sentidos de los PMG reconstruidos a partir del análisis del relato de los profesionales entrevistados (i) Fuente de modernidad y eficiencia pero con tensiones en su implementación; (ii) mecanismo de control e intensificación del trabajo; (iii) herramienta valorada y naturalizada de mejora de renta; (iv) origen de nuevas exigencias en términos de competencias y capacidades genéricas; (v) herramienta que aleja del nivel local de los servicios la definición y evaluación de los objetivos del trabajo; (vi) lógica de organización que bloquea la creatividad colectiva e innovación; (vii) herramienta que mecaniza y lleva a una pérdida de sentido del trabajo público; (viii) instrumento que homogeniza y no reconoce particularidad de cada servicio ni del trabajo dentro del Estado- permiten una reconstrucción de sus experiencias de trabajo en el contexto de la NGP.

Si bien se trata de un estudio exploratorio, con una muestra sumamente pequeña y focalizada en un solo servicio público del Estado de Chile, los hallazgos ofrecen ciertas luces sobre el impacto de los PMG a nivel de las experiencias de trabajo vistos desde la perspectiva de los propios trabajadores. Se trata de sentidos que si bien reconocen aspectos valiosos de los PMG en términos de herramienta de gestión que permite modernizar el trabajo público, al mismo tiempo que mejorar los niveles de renta en el Estado, también dan cuenta de aspectos ambivalentes o más problemáticos para la experiencia cotidiana de trabajo. Esos aspectos son consistentes con lo indicado por otros estudios nacionales e internacionales respecto a los impactos negativos y precarizantes de las reformas gerenciales de la Nueva Gestión Pública, especialmente de aquellas con un fuerte énfasis neomanagerial que desconocen la especificidad de los bienes y del trabajo público (Bryson et al, 2014; Denhardt \& Denhardt, 2000; Guerrero, 2009; O 'Flynn, 2007; Pliscoff-Varas, C. ,2017; Siltala, 2013)

Los ocho sentidos reconstruidos dan cuenta de experiencias de trabajo en el marco de la NGP y de instrumentos de gestión como los PMG que tienen un carácter tensionado y ambivalente. Al respecto son tres las principales tensiones que pensamos se pueden identificar a partir del análisis de los sentidos reconstruidos. 
En primer lugar, una tensión entre una lógica organizativa que exige y demanda a los equipos profesionales una capacidad de autonomía, creatividad y toma de decisión, al mismo tiempo que configura un proceso de trabajo con altos niveles de estandarización y formalización en torno a indicadores, responsabilización individual y con recursos insuficientes. Aparece un estado de bloqueo de la capacidad de creación y pensamiento colectivo, en el marco de procesos altamente racionalizados que sustraen a los trabajadores la posibilidad de definir y evaluar más localmente sus objetivos de trabajo. Ello produce una experiencia de malestar e insatisfacción como lo ilustran los distintos testimonios presentados.

En segundo lugar, una tensión entre un proceso de trabajo que exige una alta coordinación funcional e hiperconectividad de distintos equipos, unidades y trabajadores individuales, pero que al mismo tiempo promueve una lógica de trabajo individualizado, solitario y con escasos espacios colectivos de colaboración y reconocimiento entre colegas. La intensificación del trabajo, la vigilancia entre pares para registrar y reportar datos, así como para cumplir las metas y conseguir los bonos que se asumen como parte de la remuneración, la falta de tiempo dada la presión de resultados y la insuficiencia de recursos, lleva a orientaciones hacia el trabajo de carácter más defensivo, basadas en el temor, inhibición y vigilancia, y donde cada individuo parece refugiarse en su propia zona de tareas propias.

En tercer lugar, se aprecia una tensión entre los discursos de la NGP que relevan la gran importancia de una gestión pública moderna, eficiente, transparente y al servicio de las personas, y el despliegue de formas de organización del trabajo que erosionan el sentido y la ética del servicio público. La instalación de una lógica clientelar entre distintas unidades y con los usuarios de los servicios, las dinámicas de evaluación permanente, el temor a perder el empleo, la fuerte regulación de las tareas a partir de la definición de metas a alcanzar, la hiperespecialización en los sistemas e instrumentos de gestión, entre otros, favorece una experiencia de trabajo mecanizado, desimplicado, que no ofrece con claridad posibilidades de aprendizaje, desarrollo o cultivo de una cierta vocación. Todo ello va socavando la motivación y el horizonte ético al interior del trabajo público.

Los relatos de los profesionales entrevistados dan cuenta, al hablar de su trabajo y los PMG, de una experiencia laboral marcada por ese campo de tensiones, lo que tiene implicancias problemáticas tanto a nivel del bienestar psicosocial de los trabajadores como de la eficiencia, ética y calidad del trabajo público en el Estado. Por cierto, existen variaciones entre los profesionales, siendo los trabajadores mayores quienes tienden a tener una visión más crítica de estas tensiones dado el recuerdo nostálgico de otras formas de trabajo y organización que conocieron dentro del mismo Estado. Por el contrario, los más jóvenes tienden a naturalizar y ajustarse más pragmáticamente a las exigencias y competencias de las nuevas lógicas de gestión, dando cuenta de una transformación progresiva de los referentes de sus identidades socio-profesionales (Stecher, \& Soto, 2019). Asimismo, los resultados indican variaciones entre los profesionales de áreas de la economía e ingeniería, y aquellos de las ciencias sociales, siendo que estos últimos reportarían mayores experiencias de malestar laboral y visiones más críticas respecto a las consecuencias de los PMG. Asimismo, es importante destacar que las experiencias laborales de los profesionales no se reducen en ningún caso al vínculo con los 
PMG y que estas son más amplias e incluyen también diversas fuentes de satisfacción y reconocimiento, las cuales fueron menos recogidas en las entrevistas y relevadas en el análisis, dado el foco acotado y específico del estudio.

Con todo, y como lo han señalado otros estudios críticos respecto a los procesos de managerialización e instrumentación del trabajo público que instaló la NGP en Chile (Palacios, Hidalgo, Cornejo \& Suárez, 2019; Pliscoff-Varas, 2017; Sisto, 2012; Soto, Fardella, Valenzuela \& Carvajal, 2017), los resultados dan cuenta de ciertas tendencias problemáticas a nivel de las experiencias laborales de los profesionales del Estado producto de la implementación de los PMG. Se trata de tendencias que dan cuenta no sólo de lógicas de precarización del empleo (vínculo contractual) en el sector público -tema ampliamente estudiado y discutido-, sino de dinámicas de precarización de las experiencias de trabajo producto de los nuevos sistemas y modelos de gestión gerencial y neomanagerial (Stecher \& Sisto, 2019). Si bien la NGP no se reduce a los PMG, existiendo evaluaciones más positivas de otras dimensiones de dichas reformas en Chile -como es el caso de la creación del Sistema de Alta Dirección Pública- no es menos cierto que dicha herramienta de gestión ha sido un pilar fundamental de la gestión presupuestaria vinculada a resultados y ha tenido un impacto profundo en la reorganización del trabajo público (Morales, 2014)

En el marco del debate nacional e internacional actual sobre la necesidad de una nueva agenda y nuevas reformas a la Gestión Pública que permitan superar las limitaciones presentes en las lógicas gerenciales y neomanageriales más extremas de la NGP - como ha sido planteado en propuestas tales como "Join Up
Government" (Ling, 2002), “Whole-of-Government” (Christensen \& Lægreid 2007), Gobernanza (Aguilar, 2010), Gobierno abierto (CEPAL/ILPES, 2018), New Public Service (Denhardt \& Denhardt, 2000), Public Value Management (O'Flynn, 2007)- escuchar y analizar las voces y experiencias de los trabajadores de lo público resulta fundamental.

El presente artículo, a partir de un estudio de caso de carácter exploratorio y muy acotado, espera aportar desde la Psicología Social del Trabajo a dicha reflexión y comprensión del trabajo en el Estado y de los actuales desafíos de la gestión pública, visibilizando las experiencias de los propios actores laborales. Ese conocimiento psicosocial que recupera las perspectivas y sentidos de los propios trabajadores de lo público ha sido menos desarrollado en los estudios y debates sobre la administración del Estado, y resulta fundamental para una comprensión compleja del fenómeno, así como para contribuir y generar propuestas que sean un aporte a las unidades de gestión y desarrollo de las personas dentro del Estado.

\section{AGRADECIMIENTOS}

Este artículo ha contado con el financiamiento y/o apoyo de los proyectos: (i) ANID-FONDECYT Regular 1171088, y (ii) ANID-Programa Iniciativa Científica Milenio, Código NCS17_007-Núcleo “Autoridad y Asimetrías de Poder". El artículo está basado en los resultados de la Tesis de Magíster “Significados de los programas de mejoramiento de gestión (PMG) en profesionales del aparato público en chile. aportes a la comprensión de las experiencias de trabajo bajo la nueva gestión pública" elaborada por María Isabel Leal Llanos para optar al grado de Magíster en Psicología, Mención Psicología Social, de la Universidad Diego Portales. 


\section{Declaración de conflicto de interés}

Los autores no declaran ningún conflicto de interés.

\section{REFERENCIAS}

Aguilar, L. F. (2010). Gobernanza: El nuevo proceso de gobernar. México City: Fundación Friedrich Naumann para la Libertad.

Arellano-Gault, D. (2002). Nueva Gestión Pública: ¿el meteorito que mató al dinosaurio? Lecciones para la reforma administrativa en países como México. Revista del CLAD Reforma y Democracia, (23).

Bresser Pereira, L. C. (2002). Reforma de la Nueva Gestión Pública: Ahora en la Agenda de América Latina, Documentos y Aportes en Administración Pública y Gestión Estatal, (3), 41-64. doi:10.14409/da.v1i3.1174

Bryson, J. M., Crosby, B. C., \& Bloomberg, L. (2014). Public Value Governance: Moving Beyond Traditional Public Administration and the New Public Management. Public Administration Review, 74(4), 445-456. doi:10.1111/puar.12238

Banco Mundial. 2008. “Chile: Estudio de la Evaluación en Profundidad del Programa de Mejoramiento de la Gestión (PMG.) http://www.dipres.cl/574/articles42963 doc pdf. pdf. Consultado el 27 de octubre de 2018.

CEPAL (Comisión Económica para América Latina y el Caribe) e ILPES (Instituto Latinoamericano y del Caribe de Planificación Económica y Social) (2018), Panorama de la Gestión Pública en América Latina y el Caribe. Un gobierno abierto centrado en el ciudadano. Santiago: CEPAL-Naciones Unidas

Christensen, T., \& Lægreid, P. (2007). The Whole-ofGovernment Approach to Public Sector Reform. Public Administration Review, 67(6), 1059-1066. doi:10.1111/j.1540-6210.2007.00797.x

CLAD (Centro Latinoamericano de Administración para el Desarrollo) (1998), Una Nueva Gestión Pública para América Latina. CLAD.

Corbin y Strauss. (1998). Bases de la investigación cualitativa. Técnicas y procedimientos para desarrollar la teoría fundamentada. Publicación, Inc. ISBN: 958-655-624-7 (volumen) ISBN: 958- 655-623-9 (obra completa) 
Denhardt, R. B., \& Denhardt, J. V. (2000). The New Public Service: Serving Rather than Steering. Public Administration Review, 60(6), 549-559. doi:10.1111/0033-3352.00117

Devlin, R., \& Moguillansky, G. (2010). Alianzas públicoprivadas para una nueva visión estratégica del desarrollo. Santiago: CEPAL-Naciones Unidas

Doña, K. (2006). Síntesis del proceso de modernización del Estado en Chile (1994-2003). Documentos de Apoyo Docente INAP 6: 1-33.

Dussauge Laguna, M. I. (2009a). La literatura comparada sobre reformas administrativas: Desarrollos, limitaciones y posibilidades. Gestión y política pública, 18(2), 439-495.

Dussauge Laguna, M. I. (2009b). ¿Todos los Caminos Llevan a la Nueva Gestión Pública?: tres Argumentos sobre las Reformas Administrativas en los Países en Desarrollo Transición. Estado, Gobierno y Gestión Pública, (13), 23-51. doi:10.5354/0717-6759.2009.13513

García, I. (2007). La Nueva gestión pública: evolución y tendencias. Presupuesto y gasto público (47), p 37-64.

Guerrero, 0. (2009). El fin de la nueva Gerencia

Pública. Estado, Gobierno y Gestión Pública, (13), 5-22. doi:10.5354/0717-6759.2009.13520

Gobierno de Chile. Dirección de presupuesto (2014) Programas de Mejoramiento de la Gestión PMG: Evolución y desafíos futuros. 2011-2014. ISBN: 978-956-812370-3. Fecha de acceso 29 de marzo de 2019.http:// www.dipres.gob.cl/598/articles-114720 doc pdf.

Gobierno de Chile. Dirección de presupuestos (2018), Informe Consulta Ciudadana PMG. Fecha de acceso (22 de marzo de 2019). http://www.dipres.gob.cl/598/articles-180272doc pdf.

Gobierno de Chile. Dirección de presupuestos (2021).

Programa de Mejoramiento de la Gestión (PMG).

Fecha de acceso 29 de Julio de 2021. http://www. dipres.gob.cl/598/w3-propertyvalue-15230.html

Hood, C. (1991). “A Public Management for all season?" Public Administration, 69, 3-19. doi:10.1111/j.1467-9299.1991.tb00779.x
Krause, M. (1995). La investigación cualitativa. Un campo de desafíos y posibilidades. Revista Temas de Educación,7, 19-39.

Ling, T. (2002). Delivering joined-up government in the UK: dimensions, issues and problems. Public Administration, 80(4), 615-642. doi:10.1111/1467-9299.00321

Ley 19553 de 1998. Concede asignación de modernización y otros beneficios que indica. http://www.dipres. gob.cl/598/articles-218454 doc pdf.pdf

Morales Casetti, M. (2014). Nueva Gestión Pública en Chile. Revista de Ciencia Política (Santiago), 34(2), 417-438. doi:10.4067/s0718-090x2014000200004

O’Flynn, J. (2007). From New Public Management to Public Value: Paradigmatic Change and Managerial Implications. Australian Journal of Public Administration, 66(3), 353-366. doi:10.1111/j.1467-8500.2007.00545.x

Osborne, D. \& Gaebler, T. (1992), Reinventing Government: How the Entrepreneurial Spirit is Transforming the Public Sector. New York: Addison-Wesley

Palacios Díaz, D., Hidalgo Kawada, F., Cornejo Chávez, R., \& Suárez Monzón, N. (2019). Análisis Político de Discurso: Herramientas conceptuales y analíticas para el estudio crítico de políticas educativas en tiempos de reforma global. Education Policy Analysis Archives, 27, 47. doi:10.14507/epaa.27.4269

Pliscoff, C. \& Araya, J. P. (2012), Las alianzas público-privadas como gatilladoras de innovación en las organizaciones públicas: reflexiones a partir de la situación chilena. Revista Estado, Gobierno y Gestión Pública, 19, 173 - 198. doi:10.5354/0717-6759.2012.21180

Pliscoff-Varas, C. (2017). Implementando la nueva gestión pública: problemas y desafíos a la ética pública. El caso chileno. Convergencia Revista de Ciencias Sociales, (73). doi:10.29101/crcs.v0i73.4241

Ramírez Alujas, Á. (2004). El proceso de reforma del Estado y modernización de la gestión pública en Chile. Lecciones, experiencias y aprendizajes (1990-2003). Instituto Nacional de Administración Pública (INAP). Resultados Programa de Mejoramiento de la Gestión PMG, ISBN: 978-9569931-25-3, Agosto 2018, Editorial creativo Chile. 
Ruiz, J.I. (2003). Metodología de la investigación cualitativa. Bilbao:Universidad de Deusto.

Siltala, J. (2013), New Public Management: The EvidenceBased worst practice? Administration \& Society. 45(4), 468 - 493. doi: 10.1177/0095399713483385

Soto, A., Fardella, C., Valenzuela, A., \& Carvajal, F. (2017). La funciones performativas de los instrumentos en las redes de acción pública. Psicoperspectivas. Individuo y Sociedad, 16(3). doi:10.5027/ psicoperspectivas-vol16-issue3-fulltext-999

Stecher, A. (2010). El análisis crítico del discurso como herramienta de investigación psicosocial del mundo del trabajo: discusiones desde América Latina. Universitas Psychologica, 9(1), 93-107. doi:10.11144/javeriana.upsy9-1.acdh

Stecher, A., Soto, A. (2019) Crisis and Transformation of Occupational Identities in Three Sectors (Retail, Mining, State): Contributions to Understanding Workplace Subjectivities in Neoliberal Chile. Subjectivity, 12(4), 309-332 https://doi.org/10.1057/s41286-019-00080-x

Stecher, A. y Sisto, V. (2019) Trabajo y precarización laboral en el Chile neoliberal. Apuntes para comprender el estallido social. En Araujo, K. (edit). Hilos tensados. USACH.

Thompson, J.B. (1998). Ideología y cultura moderna. México, DF: Universidad Autónoma Metropolitana 